\title{
Avaliação dos parâmetros de calcinação do resíduo de gesso nas propriedades do gesso reciclado
}

\section{Evaluation of the calcination parameters of the gypsum waste on the properties of recycled plaster}

Pâmela Bento Cipriano ${ }^{1}$, Tássila Saionara Gomes Galdino ${ }^{1}$, Clebiano Santos Sá ${ }^{1}$, Andréa de Vasconcelos Ferraz ${ }^{1}$

\footnotetext{
${ }^{1}$ Universidade Federal do Vale do São Francisco (UNIVASF), Pós-Graduação em Ciências dos Materiais, Av. Antônio C. Magalhães, 510, Country Club, CEP: 48902-300, Bahia, Juazeiro, Brasil.

e-mail: pamela.ufcg@gmail.com, tassilasaionara@gmail.com, clebiano@hotmail.com, andrea.ferraz@univasf.edu.br
}

\section{RESUMO}

As atividades industriais têm contribuído com a geração de resíduos de gesso, logo o alto volume e a destinação inapropriada contribuem com os problemas ambientais, além de representar desperdício de material na construção civil. O objetivo do trabalho foi caracterizar o resíduo de gesso e avaliar a influência de parâmetros como granulometria, temperatura e tempo de calcinação nas propriedades do gesso reciclado, buscando a redução dos resíduos e obtenção de melhores propriedades do produto final com a utilização de $100 \%$ de gesso reciclado. $\mathrm{O}$ resíduo de gesso foi beneficiado, caracterizado e separado em três diferentes granulometrias: peneira $\mathrm{ABNT}^{\circ} 200(0,075 \mathrm{~mm})$; $\mathrm{ASTM} \mathrm{n}^{\circ} 50(0,3 \mathrm{~mm})$ e $\mathrm{ABNT}^{\circ} 10(2,0 \mathrm{~mm})$. Em seguida calcinados nas temperaturas de $130,136,150$ e $170^{\circ} \mathrm{C}$ durante $1,2,4,6,8,17$ e $24 \mathrm{~h}$. Foi analisada no gesso reciclado a sua trabalhabilidade, calor de hidratação, propriedades mecânicas, caracterização mineraló gica e morfologia. O gesso reciclado teve influência nas suas propriedades nos parâmetros avaliados, além da presença de impurezas. As principais fases formadas no gesso reciclado foram afetadas pelo tempo de calcinação: o sulfato de cálcio hemihidratado, sulfato de cálcio dihidratado, anidrita e calcita. A trabalhabilidade não mostrou uma tendência clara de comportamento independente da granulometria, tempo e temperatura de calcinação. Os melhores resultados para a resistência compressão foram obtidos na temperatura de $136^{\circ} \mathrm{C}(17 \mathrm{~h})$ e $150^{\circ} \mathrm{C}(8 \mathrm{~h})$. O gesso reciclado apresentou uma dureza média de $12,71 \mathrm{~N} \cdot \mathrm{mm}^{-2}$. A reciclagem do resíduo de gesso pode contribuir com a redução dos resíduos e possibilitar na construção civil a utilização de novas tecnologias.

Palavras-chave: resíduo de gesso; reciclagem; granulometria; calcinação.

\begin{abstract}
Industrial activities have contributed to the generation of gypsum waste, so the high volume and inappropriate disposal contributes to environmental problems and represents waste of material. The objective of this work was to characterize the waste of gypsum and to evaluate the influence of parameters such as granulometry, temperature and calcination time on the properties of the recycled gypsum, seeking the reduction of residues and obtaining better properties of the final product with the use of $100 \%$ of recycled plaster. The gypsum waste was beneficiated, characterized and separated into three different granulometries: sieve ABNT $\mathrm{n}^{\circ} 200(0.075 \mathrm{~mm})$; ASTM $\mathrm{n}^{\circ} 50(0.3 \mathrm{~mm})$ and ABNT $\mathrm{n}^{\circ} 10(2.0 \mathrm{~mm})$. They were then calcined at temperatures of $130,136,150$ and $170^{\circ} \mathrm{C}$ for $1,2,4,6,8,17$ and $24 \mathrm{~h}$. Its workability, heat of hydration, mechanical properties, mineralogical characterization and morphology were analyzed in the recycled plaster. The recycled gypsum was influenced in its properties in the evaluated parameters, besides the presence of impurities. The main phases formed in the recycled gypsum were affected by the calcination time: calcium sulfate hemihydrate, calcium sulfate dihydrate, anhydrite and calcite. The workability did not show a clear trend of behavior independent of grain size, time and calcination temperature. The best results for the compressive strength were obtained at the temperature of $136^{\circ} \mathrm{C}(17 \mathrm{~h})$ and $150^{\circ} \mathrm{C}(8 \mathrm{~h})$. The recycled plaster had an aver-
\end{abstract}


age hardness of $12.71 \mathrm{~N} \cdot \mathrm{mm}^{-2}$. The recycling of gypsum waste can contribute to the reduction of waste and make possible the use of new technologies in civil construction.

Keywords: waste of gypsum; recycling; granulometry; calcination.

\section{INTRODUÇÃO}

O gesso pode ser usado na forma natural com crescimento intenso na construção civil e calcinado amplamente utilizados em edifícios, cerâmica e indústrias médicas [1-3]. Geralmente o resíduo de gesso está disponível em forma de pó, torrões ou pedaços provenientes de produtos como chapas para drywall e placas, logo para a sua reutilização é necessário triturar, moer e peneirar [4].

Aspectos como o consumo de energia para sua fabricação e a alta geração de resíduos de gesso nas atividades da construção civil causam problema econômico e ao meio ambiente [5]. O alto volume e a destinação inapropriada dos resíduos de gesso possibilitaram na construção civil a utilização de novas tecnologias ou a adaptação de materiais antigos à nova visão de mercado, visando redução de custo e sustentabilidade [6].

$\mathrm{O}$ desenvolvimento de materiais inovadores e sustentáveis tem se tornado prioridade para muitos pesquisadores principalmente para materiais poluentes ao meio ambiente. A utilização de resíduo de gesso representa uma importante fonte de matéria prima na construção civil proporcionando economia e redução do impacto ambiental $[7,8]$. O gesso reciclado derivado de fontes pós-consumo é interessante para ser reincorporado na cadeia de produção, devido ao custo ser menor do que o gesso convencional [9]. Algumas empresas em Pernambuco estão treinando os gesseiros para reciclar o resíduo gerado do próprio revestimento, adicionando até $20 \%$ do resíduo seco ou úmido à mistura sem nenhum tipo de critério [10].

Para a reutilização de resíduos de gesso é muito utilizado o processo de reciclagem através da secagem do resíduo por meio de uma reação reversível do dihidrato em hemihidrato de gesso [11]. Estudo indica a possibilidade da reciclagem do gesso com baixo consumo de energia. Foi avaliado o tempo de calcinação de resíduos de gesso para a produção de gesso com propriedades aglutinantes para uso em placas de gesso. A calcinação foi realizada a $150^{\circ} \mathrm{C}$ durante $3,4,5$ e $6 \mathrm{~h}$. Os melhores resultados para resistência a compressão foi obtido no período de 5 e $6 \mathrm{~h}$ e dureza $3,4,5$ e $6 \mathrm{~h}$. No estado fresco o material reciclado apresentou bons resultados para os componentes pré-moldados [12].

Os autores mostraram a análise de dois tipos diferentes de resíduos de gesso em substituição ao gesso comercial: resíduos de gesso da produção industrial de gesso acartonado e gesso de dessulfuração de gases de combustão a partir de uma central térmica. Avaliou-se a influência do processo de calcinação nos resíduos de gesso sendo submetidos as condições: temperatura de $100^{\circ} \mathrm{C}$ por 6 e $24 \mathrm{~h}$, e a $150^{\circ} \mathrm{C}$ durante 3 e $6 \mathrm{~h}$. O melhor resultado na transformação de todo o gesso dihidratado em bassanite (hemihidrato) foi a temperatura de calcinação de $150^{\circ} \mathrm{C}$ durante $3 \mathrm{~h}[13]$.

Durante a calcinação do resíduo de gesso passa por uma desidratação em estufa a uma determinada temperatura e por um período de tempo para obtenção do gesso reciclado (hemihidrato) [14]. Os produtos da desidratação do gesso podem ser hemihidrato e anidritas, vários fatores podem influenciar nas reações como o tipo de gesso e condições ambientais resultando em variações nas temperaturas de transição [15]. Levando em consideração a grande quantidade de fatores que podem influenciar nas propriedades do gesso reciclado justifica a importância do estudo para visabilizar o reaproveitamento do resíduo de gesso.

O objetivo do trabalho foi caracterizar o resíduo de gesso e avaliar a influência dos parâmetros de calcinação como granulometria, temperatura e tempo nas propriedades do gesso reciclado. Permitindo a reciclagem do resíduo de gesso contribuindo com a redução dos resíduos e obtenção de melhores propriedades do produto final com a utilização em maior escala do gesso reciclado.

\section{MATERIAIS E MÉTODOS}

O resíduo de gesso foi obtido da fratura dos produtos acabados no processo produtivo de blocos e placa de gesso em uma empresa de pré-moldados localizada no município de Juazeiro-BA.

O beneficiamento do resíduo para obtenção da granulometria adequada para a caracterização, similar à do gesso de fundição, foi realizada por via seca em moinho de bolas da marca Pavitest, modelo I-3021, utilizando bolas de ferro, por um período de $4 \mathrm{~h}$.

\subsection{Caracterização do resíduo de gesso}

A caracterização mineralógica do resíduo de gesso e do gesso reciclado foi realizada através da difração de Raios X (DRX), utilizou-se um difratômetro marca Shimadzu (XRD-6000), operando com radiação k-alfa de cobre $(\lambda=0,15406 \mathrm{~nm}), 2 \theta$ variando de 5 a $90^{\circ}$ e uma velocidade de varredura de $2^{\circ} \cdot \mathrm{min}^{-1}$, operando a $30 \mathrm{kV}$ e $30 \mathrm{~mA}$ na temperatura ambiente. A análise dos difratogramas foram realizados utilizando o software X'Pert 
HighScore Plus versão 2.0a e os padrões das cartas cristalográficas do PDF2-ICSD (2003).

A composição química do resíduo de gesso foi obtida após o beneficiamento no moinho de bolas por fluorescência de Raios X (FRX) em equipamento da marca EDX 720 (Shimadzu). A matéria orgânica foi determinada de acordo com a metodologia da Embrapa [16] pelo Laboratório de Análises de Solo e Planta (LASP) em Petrolina-PE.

Os grupos funcionais presentes no resíduo foram determinados por Infravermelho com Transformada de Fourrier (FTIR) em um aparelho Spectrum Two (Perkin Elmer), com varredura de 4000 a $400 \mathrm{~cm}^{-1}$. As amostras na forma de pó foram prensadas em pastilhas com $30 \mathrm{mg}$ de $\mathrm{KBr}$ previamente seco.

A caracterização física do pó do resíduo de gesso foi realizada através do ensaio granulométrico e de massa unitária de acordo com a norma NBR 12127 [17], comparando os resultados com os requisitos do gesso para a construção civil através descrito na norma NBR 13207 [18].

O comportamento térmico do resíduo foi analisado através de um equipamento de análise simultânea DTA-TG (Análise térmica diferencial/Termogravimetria) da marca Shimadzu (DTG-60H). A análise foi realizada em cadinho de alumina com massa de aproximadamente $10 \mathrm{mg}$ sob atmosfera de nitrogênio com fluxo do gás de $100 \mathrm{~mL} \cdot \mathrm{min}^{-1}$, com taxa de aquecimento $10^{\circ} \mathrm{C} \cdot \mathrm{min}^{-1} \mathrm{e}$ temperatura de 25 até $1000^{\circ} \mathrm{C}$.

A análise morfológica do pó e da pasta do resíduo de gesso que foi preparada com razão de água 0,8 determinada no ensaio de consistência normal, foram realizadas por um microscópio eletrônico de varredura da marca Tescan (Vega 3XMU), com magnificações de 4.000×. As amostras foram recobertas com ouro durante 10 min e corrente de $15 \mathrm{~mA} \cdot 5 \mathrm{~min}^{-1}$, utilizando um equipamento da marca Quorum (Q150R ES).

\subsection{Calcinação do resíduo de gesso}

Para realizar a calcinação o resíduo de gesso foi separado em três diferentes granulometrias: peneira ABNT $\mathrm{n}^{\circ} 200(0,075 \mathrm{~mm})$; ASTM n $50(0,3 \mathrm{~mm})$ e ABNT no $10(2,0 \mathrm{~mm})$. Em seguida, cada granulometria foi calcinada nas temperaturas de $130,136,150{ }^{\circ} \mathrm{C}[5]$ e $170^{\circ} \mathrm{C}$ durante $1,2,4,6,8,17$ e $24 \mathrm{~h}$.

\subsection{Avaliação do gesso reciclado}

A avaliação da trabalhabilidade do gesso reciclado após a calcinação em diferentes condições foi realizado conforme a norma DIN 1168 [19].

Utilizando um calorímetro pseudoadiabático, obteve-se a curva do calor de hidratação em função do tempo, onde foram medidos os tempos de pega das amostras. O tempo de início de pega foi caracterizado quando a temperatura variou $0,1^{\circ} \mathrm{C} \cdot \mathrm{min}^{-1}$. $\mathrm{O}$ de fim de pega foi obtido quando a temperatura máxima foi atingida.

Para a caracterização da pasta, o resíduo foi passado pela peneira ASTM n ${ }^{\circ} 50(0,29 \mathrm{~mm})$ e calcinado por um período de $8 \mathrm{~h}$ a temperatura de $136^{\circ} \mathrm{C}$, a qual foi definida através da análise térmica. A avaliação dos tempos de início e fim de pega, e consistência normal do resíduo de gesso foi determinada pela NBR 12128 (2017).

Os corpos de prova do gesso reciclado para análise das propriedades mecânicas de dureza e compressão foram preparados de acordo com a norma NBR 12129 [20] utilizando a razão água/gesso determinado no ensaio de consistência normal.

Os ensaios de resistência mecânica à compressão foram realizados em uma máquina universal de ensaios mecânicos (Emic-DL 10000) e o ensaio de dureza em equipamento projetado por LÊLA [21] de acordo com a norma de propriedades mecânicas para gesso NBR 12129 [20].

As micrografias das pastas preparadas com o gesso reciclado foram obtidas em um estereoscópio da marca Marte usando luz refletida.

\section{RESULTADOS}

\subsection{Caracterização do resíduo de gesso}

A composição química do resíduo de gesso (Tabela 1) foi determinada após a etapa de moagem e foi observada a presença de cálcio, enxofre, fósforo, silício, estrôncio e matéria orgânica.

Os elementos cálcio e enxofre representam 98,49\% e um teor de aproximadamente 1,50\% de impurezas da massa do resíduo. Composto principalmente por sulfato de cálcio dihidratado $\left(\mathrm{CaSO}_{4} \cdot 2 \mathrm{H}_{2} \mathrm{O}\right)$, e as impurezas apresentadas depende da fonte do resíduo [7]. Resultados reportados na literatura mostram em resíduo de gesso um teor menor que $1 \%$ para impurezas como: $\mathrm{SrO}, \mathrm{SiO}_{2}, \mathrm{Al}_{2} \mathrm{O}_{3}, \mathrm{Fe}_{2} \mathrm{O}_{3}, \mathrm{PbO}, \mathrm{CuO}$ e $\mathrm{MgO}$ [22]. A presença de impurezas pode aumentar o tempo de pega e reduzir as propriedades mecânicas do gesso reciclado. 
No resíduo de gesso analisado foi encontrado a presença de fósforo, na literatura foram identificados no gesso $\beta$ pequenos traços de fósforo [23]. Foi observada uma baixa quantidade de matéria orgânica.

Tabela 1: Composição química do resíduo de gesso.

\begin{tabular}{ccccccc}
\hline \multirow{2}{*}{ Amostra } & \multicolumn{5}{c}{ Teores dos elementos (\%) } & \multirow{2}{*}{ Matéria orgânica $\left(\mathbf{g} \cdot \mathbf{k g} \mathbf{~}^{\mathbf{1}}\right)$} \\
\cline { 2 - 6 } & $\mathbf{C a}$ & $\mathbf{S}$ & $\mathbf{P}$ & $\mathbf{S i}$ & $\mathbf{S r}$ & \\
\hline Resíduo de gesso & 65,18 & 33,31 & 0,85 & 0,46 & 0,20 & 0,20 \\
\hline
\end{tabular}

Na Figura 1 são apresentados os grupos funcionais característicos do resíduo de gesso, antes da calcinação, obtidos por FTIR. As bandas do grupo O-H presentes em 3546,89; 3401,96 e 2234,12 cm são refe- $^{-1}$ rentes às ligações das moléculas de água. Foram identificadas também bandas do grupo sulfato $\left(\mathrm{SO}_{4}^{-2}\right)$ em $1143,31 \mathrm{e} 453,74 \mathrm{~cm}^{-1}$ [23]. Os grupos funcionais são característicos do resíduo de gesso $\left(\mathrm{CaSO}_{4} \cdot 2 \mathrm{H}_{2} \mathrm{O}\right)$. Os autores CARVALHO et. al [24] mostraram que a curva do gesso reciclado é semelhante à do gesso comercial. Portanto, após o processo de desidratação por calcinação o gesso reciclado mantém a estrutura dos principais grupos funcionais do resíduo de gesso.

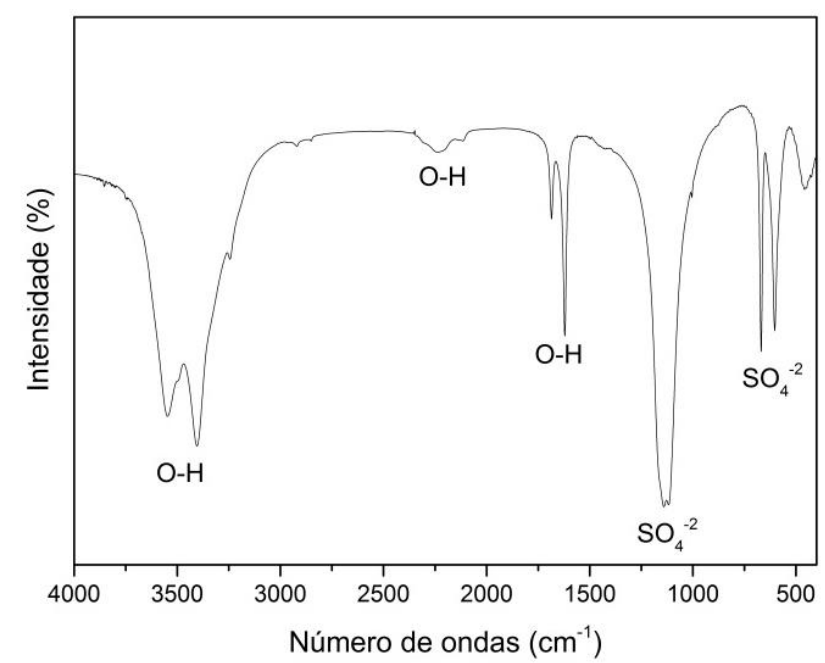

Figura 1. Espectro de Infravermelho do resíduo de gesso.

Os resultados obtidos para a massa unitária e a granulometria são mostrados na Tabela 2. O resíduo não atendeu a exigência da norma ao que se refere à granulometria, pois seu percentual passante na peneira $\operatorname{ASTM~n}^{\circ} 50(0,29 \mathrm{~mm})$ foi inferior aos $90 \%$ exigidos. Indicando que o método de beneficiamento utilizado não foi adequado para a redução do tamanho das partículas [25]. Resultado semelhante foi obtido em um resíduo de gesso oriundo de blocos, devido à presença de grande quantidade de grãos grossos [3].

Quanto à massa unitária o resíduo de gesso passante na peneira $\mathrm{ABNT} \mathrm{n}^{\circ} 10(2,00 \mathrm{~mm})$ atende ao requisito requerido pela norma. $\mathrm{O}$ estudo, realizado em Curitiba-PR, mostrou que a massa unitária do gesso reciclado não sofreu influência da temperatura de calcinação $\left(160,180\right.$ e $\left.200^{\circ} \mathrm{C}\right)$ [26].

Tabela 2: Resultados dos ensaios de massa unitária e granulometria.

\begin{tabular}{ccc}
\hline PROPRIEDADES & RESÍDUO DE GESSO & REQUISITO NBR 13207 (2017) \\
\hline Massa unitária $\left(\mathrm{g} \cdot \mathrm{cm}^{-3}\right)$ & $619,19 \pm 2,19$ & $\geq 600$ \\
\hline Granulometria $(\%)$ & $75,69 \pm 0,80$ & $\geq 90 \%$ passante \\
\hline
\end{tabular}

A Tabela 3 mostra os resultados obtidos pelo ensaio de Vicat e consistência conforme a norma NBR 12128 [27]. O gesso reciclado utilizado foi calcinado durante $8 \mathrm{~h} / 136^{\circ} \mathrm{C}$, essa temperatura foi definida de acordo com a análise térmica (Figura 3). A consistência normal foi obtida com a razão de água/gesso reciclado de 0,8 . O tempo de pega é um indicador que caracteriza o momento de início do endurecimento da pasta e o momento final da reação de hidratação [26]. O gesso reciclado apresentou tempo de inicio e final de pega dentro do esperado na especificação da norma NBR 13207 [18] (inicio $\leq 10$ min e final $\leq 20$ min). No entanto, a trabalhabilidade apresentou um tempo pequeno. Visando melhorar esse tempo foram avaliados diferentes temperaturas e tempos de calcinação (Figura 6). 
Tabela 3: Resultado do ensaio de Vicat e consistência.

\begin{tabular}{cccc}
\hline \multirow{2}{*}{ Razão água/gesso reciclado } & \multicolumn{2}{c}{ Tempo de pega (min) } & \multirow{2}{*}{ Consistência } \\
\cline { 2 - 3 } & Inicio & Final & \\
\hline 0,8 & $5,67 \pm 0,57$ & $9,03 \pm 0,26$ & $28,17 \pm 0,29$ \\
\hline
\end{tabular}

A Figura 2 apresenta as micrografias referentes ao resíduo de gesso em pó e a superfície de fratura dos corpos de prova preparados com o gesso reciclado a $136^{\circ} \mathrm{C}$ durante $8 \mathrm{~h}$ e razão água/gesso reciclado de 0,8 .

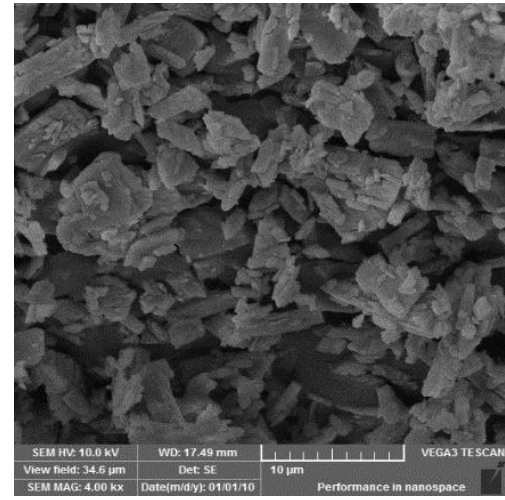

(a)

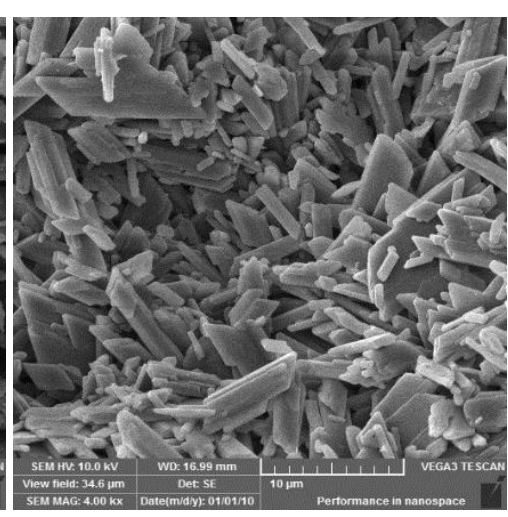

(b)

Figura 2: Micrografia de MEV com magnificação de 4000x: a) resíduo de gesso em pó e b) superfície de fratura do corpo de prova preparado com o gesso reciclado.

Foi observada na micrografia do resíduo de gesso em pó partículas com diferentes tamanhos e formatos. O gesso reciclado apresentou aglomerados de cristais alongados de tamanhos variados. Essa estrutura desenvolvida após a mistura com a água era esperada para o gesso [22]. Resultados semelhantes foram obtidos ao longo de cinco ciclos de reciclagem de gesso realizados a $180^{\circ} \mathrm{C}$ por $24 \mathrm{~h}$ [28]. A quantidade de núcleos presentes na pasta influencia a taxa de crescimento da microestrutura e o tamanho dos cristais [19]. A microestrutura do cristal formado pode aumentar com a interconexão de cristais menores e tende a reduzir a dureza da pasta de gesso no estado endurecido [11].

Figura 3 mostra a curva TG/DTA do resíduo de gesso. Na curva foi indicada a conversão do resíduo de gesso em hemihidrato no pico endotérmico em $136^{\circ} \mathrm{C}$ e em anidrita II na temperatura de aproximadamente $355^{\circ} \mathrm{C}[29]$.

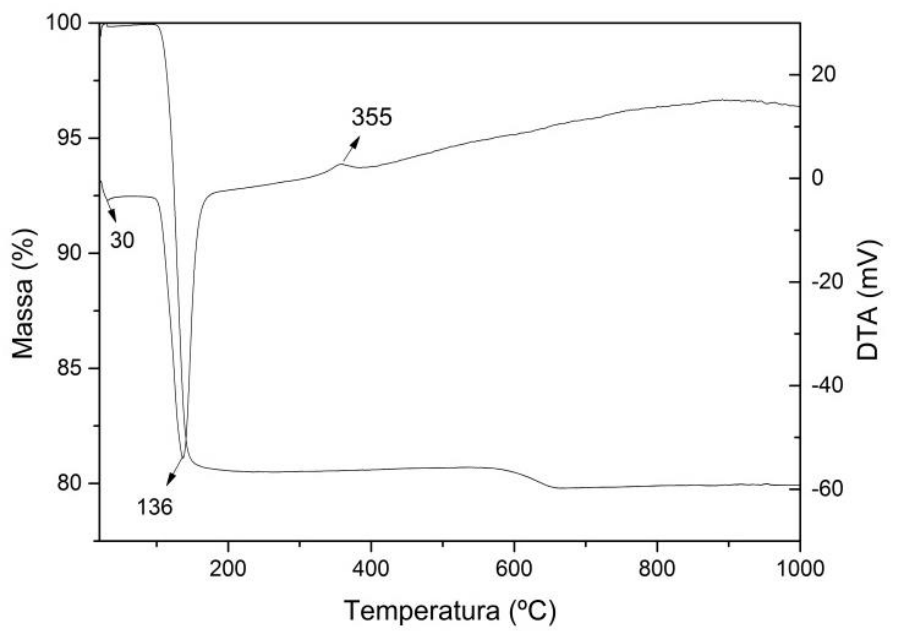

Figura 3: Curva TG/DTA do resíduo de gesso.

$\mathrm{O}$ pico endotérmico em $30^{\circ} \mathrm{C}$ está relacionado à perda de água livre, geralmente ocorre entre a temperatura ambiente até $60^{\circ} \mathrm{C}$ [5]. Observou-se um pico endotérmico em aproximadamente $136^{\circ} \mathrm{C}$ referente à perda de cerca de $20 \%$ de água estrutural do gesso [22]. O valor encontrado é semelhante ao esperado na literatura para a hidratação do gesso de construção (21\%) [30]. A curva DTA apresenta um pico exotérmico entre 350 e $360^{\circ} \mathrm{C}$ atribuído à conversão da anidrita III para anidrita II [5, 31]. 
Foi utilizado o valor do pico endotérmico principal de $136^{\circ} \mathrm{C}$ e temperaturas abaixo e acima desse valor para realizar o processo térmico de desidratação (calcinação) do resíduo de gesso para obtenção do gesso reciclado.

As reações de desidratação do gesso ou gesso reciclado apresentam as fases de desidratação quando submetidos a diferentes temperaturas [8].

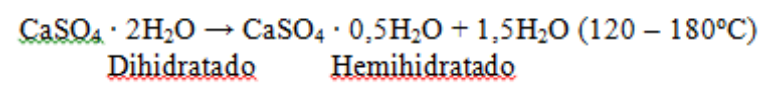
$\mathrm{CaSQ}_{4} \cdot 0,5 \mathrm{H}_{2} \mathrm{O} \rightarrow \mathrm{CaSO}_{4} \cdot \mathrm{eH}_{2} \mathrm{O}+(0,5-\varepsilon) \mathrm{H}_{2} \mathrm{O}\left(180-300^{\circ} \mathrm{C}\right)$
Hemihidratado

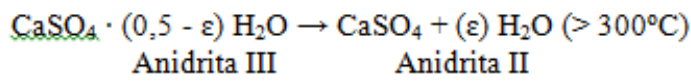

Onde, $\varepsilon$ representa o percentual não exato obtido para o gesso parcialmente desidratado o valor é inferior a 0,5 moléculas de $\mathrm{H}_{2} \mathrm{O}$. A presença das fases de sulfato de cálcio é o resultado de diferentes interações, como temperatura, pressão da água dentro do forno, distribuição de tamanho das partículas e tempo de permanência [32].

\subsection{Avaliação da calcinação do resíduo}

A Figura 4 mostra o difratograma de Raios $\mathrm{X}$ do resíduo de gesso sem calcinar e calcinado em diferentes tempos utilizando a temperatura do pico endotérmico principal $\left(136^{\circ} \mathrm{C}\right)$ obtido na curva de análise térmica (Figura 3).

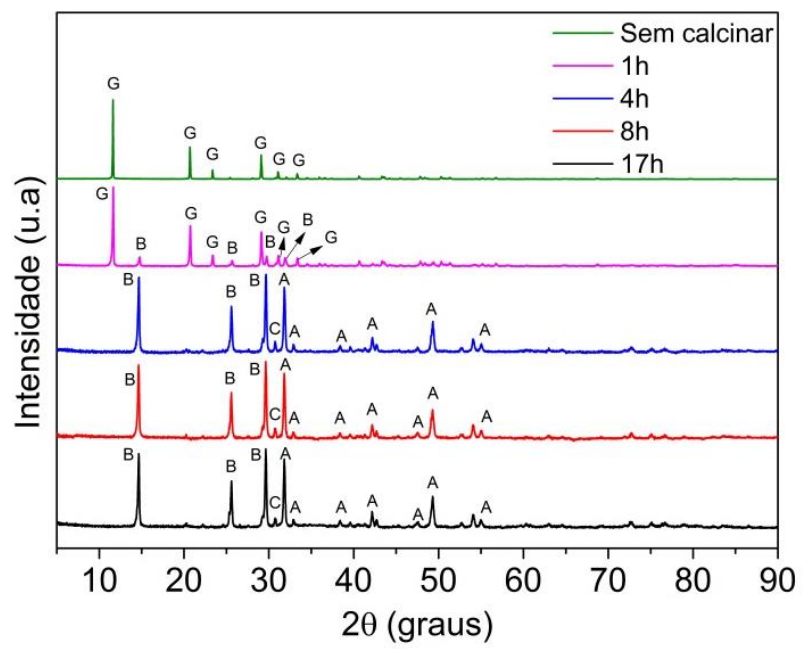

Figura 4: Difratograma de Raios $\mathrm{X}$ do resíduo de gesso $(0,30 \mathrm{~mm})$ sem calcinar e calcinado a $136^{\circ} \mathrm{C}$ durante $1,4,8$ e 17h. (G: gipsita $\left(\mathrm{Ca}\left(\mathrm{SO}_{4}\right)\left(\mathrm{H}_{2} \mathrm{O}\right)_{2}\right)$ - ICSD: 027221, B: bassanita $\left(\mathrm{CaSO}_{4} \cdot 0,5 \mathrm{H}_{2} \mathrm{O}\right)-\mathrm{PDF} 2: 00-033-0310$, A: anidrita $\left(\mathrm{Ca}\left(\mathrm{SO}_{4}\right)\right)$ - ICSD: 040043, C: calcita $\left(\mathrm{CaCO}_{3}\right)$ - PDF2: 00-003-0670).

O resíduo de gesso sem calcinar apresenta fase cristalina da gipsita. A quantidade de impurezas e de gipsita contidas no gesso acelera a reação de hidratação e o tempo de pega por atuarem como núcleos de cristalização [30]. Vale ressaltar que diferentes tipos de gesso comercial contêm diferentes quantidades de dihidrato, hemihidrato e anidrita devido à diferença na quantidade de água que é fixada aos hemihidratos após o uso do produto [33]. 
Após 1h na estufa o difratograma apresentou picos de gipsita e bassanita que corresponde ao sulfato de cálcio hemihidratado proveniente da reação de desidratação do resíduo de gesso. Foi identificada a presença de dihidrato em um gesso reciclado no primeiro ciclo calcinado a $150^{\circ} \mathrm{C}$ durante $1 \mathrm{~h}$, esse comportamento foi atribuido a etapa de secagem utilizada no processo de reciclagem. Uma grande quantidade de sulfato de cálcio hemihidrato $\left(\mathrm{CaSO}_{4} \cdot 0,5 \mathrm{H}_{2} \mathrm{O}\right)$ proporciona propriedades de ligação ao gesso reciclado, mantendo as características semelhantes ao gesso comercial. As principais fases identificadas no gesso reciclado foram: o sulfato de cálcio hemihidratado, sulfato de cálcio dihidratado e anidrita [34].

Na calcinação realizada durante 4,8 e 17 h as curvas são semelhantes, foram observados picos de bassanita, anidrita e calcita. A calcita foi identificada através do pico 2,91 ^̊ nos resíduos calcinados em tempos superiores à $4 \mathrm{~h}$, foi observada a presença desse mineral também em resíduo de gipsita oriundos de áreas de mineração no município de Araripina, Pernambuco [35]. O resíduos de gesso obtido da produção de placas apresentou calcita após a calcinação a $100^{\circ} \mathrm{C}$ por $24 \mathrm{~h}$ e na temperatura de $150^{\circ} \mathrm{C}$ durante 3 e $6 \mathrm{~h}[13]$.

O pico de anidrita identificada apresenta estrutura ortorrômbica sugerindo a formação de anidrita II, pois essa fase para ser formada depende da faixa de temperatura e tempo utilizado na calcinação. Como observado na curva de análise térmica (Figura 3) a anidrita foi determinada em $355^{\circ} \mathrm{C}$, esse comportamento era esperado na faixa de temperatura entre 310 e $450^{\circ} \mathrm{C}$ [15].

A Figura 5 mostra as micrografias das pastas preparadas com o gesso reciclado com granulometria de $0,075 \mathrm{~mm}$, calcinado na temperatura de $136^{\circ} \mathrm{C}$ permanecendo na estufa durante $1,2,4,6,8,17$ e $24 \mathrm{~h}$.

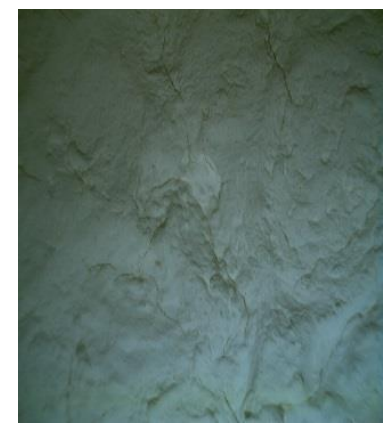

(a)

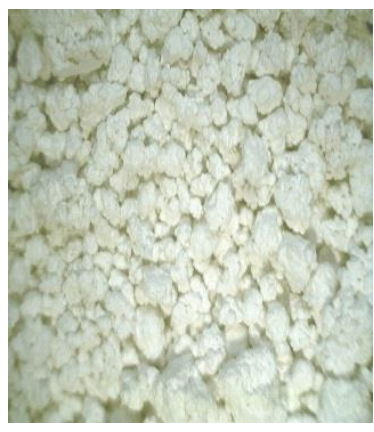

(b)

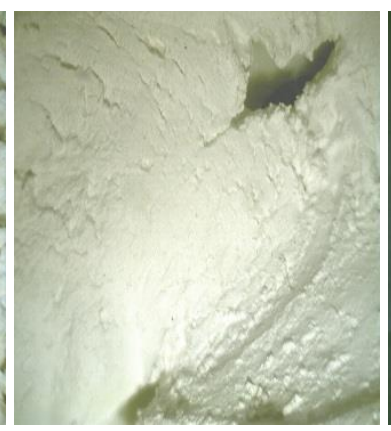

(c)

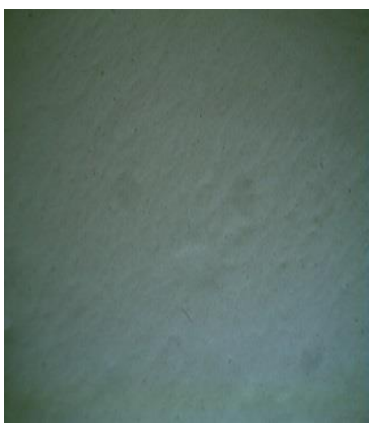

(d)

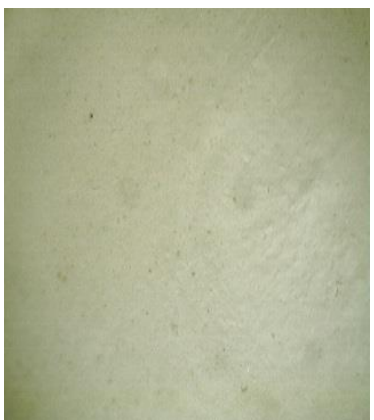

(e)

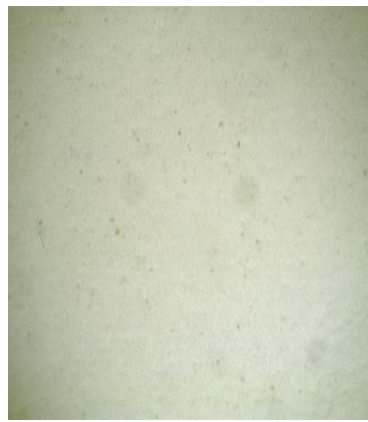

(f)

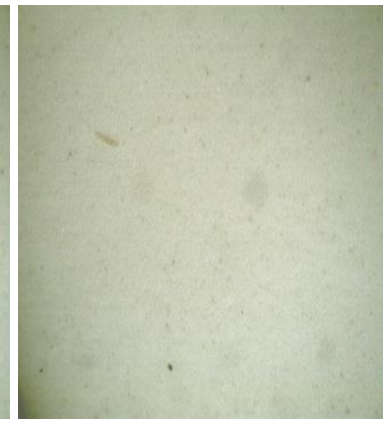

(g)

Figura 5: Micrografias obtidas da pasta de gesso reciclado com a granulometria de $0,075 \mathrm{~mm}$ calcinado a $136^{\circ} \mathrm{C}$ durante: a) $1 \mathrm{~h}$, b) $2 \mathrm{~h}$, c) $4 \mathrm{~h}$, d) $6 \mathrm{~h}$, e) $8 \mathrm{~h}$, f) $17 \mathrm{~h} \mathrm{e} \mathrm{g)} 24 \mathrm{~h}$.

Com o gesso reciclado durante $2 \mathrm{~h}$ não foi possível formar pasta, esse comportamento foi reportado na literatura como um endurecimento instantâneo ao polvilhar o gesso na água, com a formação de grumos [25]. Provavelmente, o tempo de $2 \mathrm{~h}$ de secagem na temperatura de $136^{\circ} \mathrm{C}$ não foi suficiente para desitratar o gessso e permitir uma hidratação novamente. Após 4 h de calcinação o inicio da pega foi muito rápido promovendo o endurecimento da pasta depois de ser homogeneizado. Os demais tempos avaliados foram possíveis formar a pasta.

A trabalhabilidade foi determinada para o gesso reciclado calcinado em estufa às temperaturas de 130 , 136, 150 e $170^{\circ} \mathrm{C}$ utilizando diferentes tempos e granulometrias (Figura 6). No gráfico os tempos em que a trabalhabilidade não foi representada indica que não foi possível determinar a trabalhabilidade. 


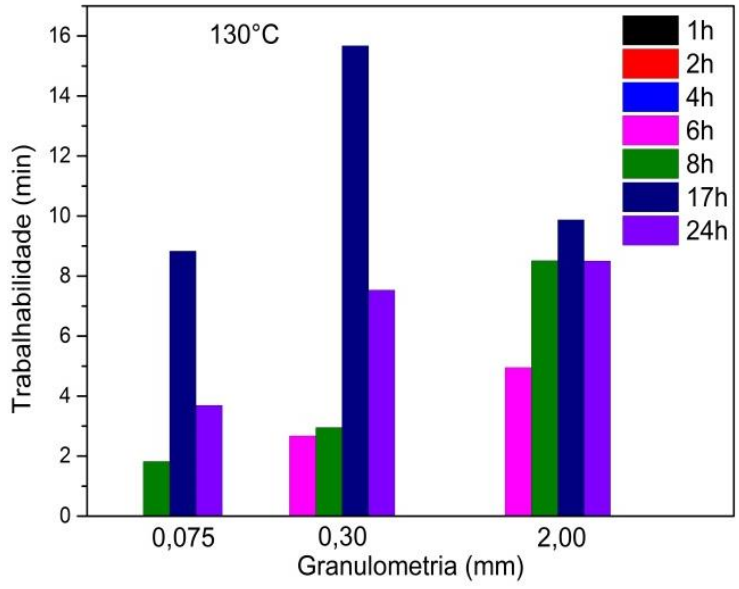

(a)

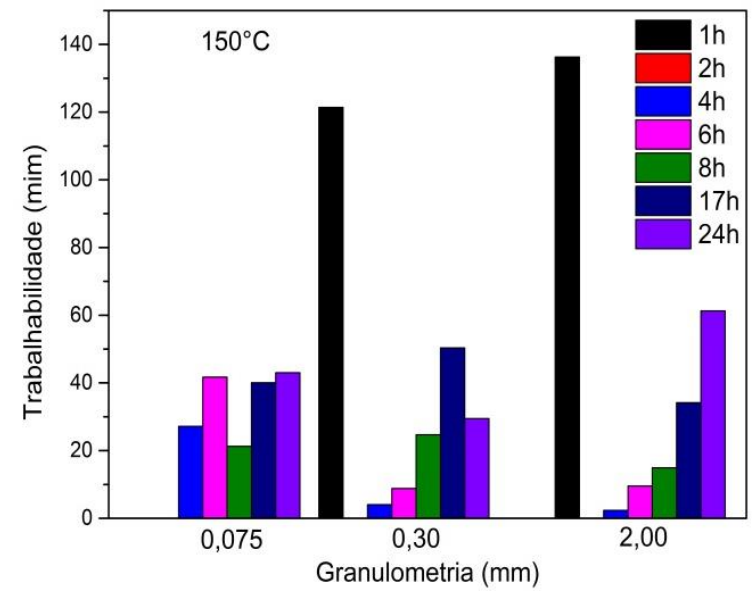

(c)

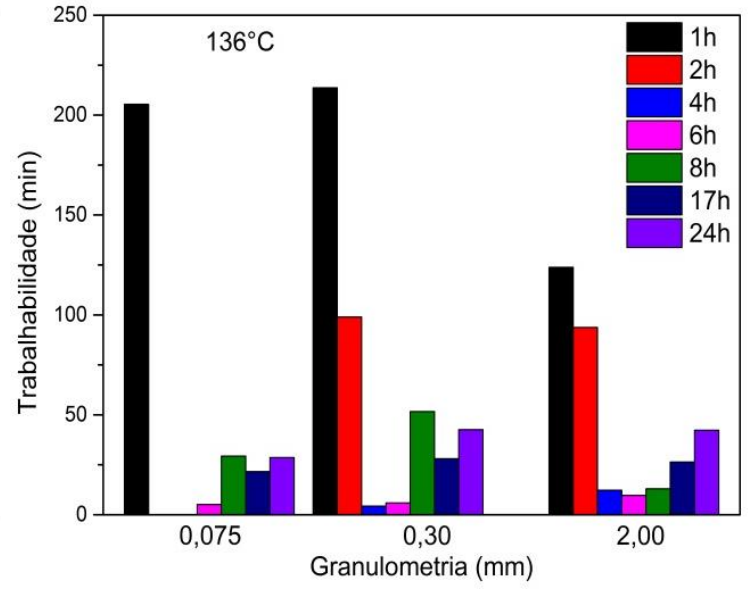

(b)

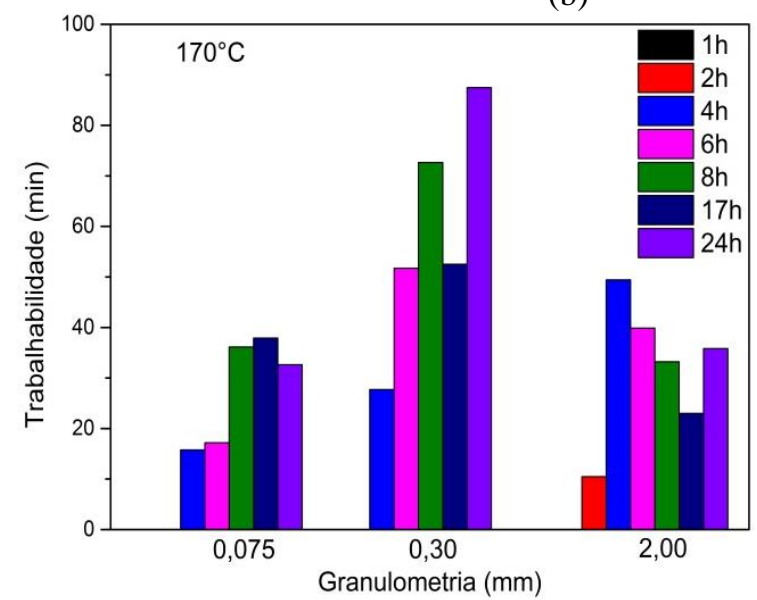

(d)

Figura 6: Trabalhabilidade do gesso reciclado obtido pela norma DIN 1168.

A trabalhabilidade não mostrou uma tendência clara de comportamento independente da granulometria, tempo e temperatura de calcinação. No gesso reciclado a $130^{\circ} \mathrm{C}$ a trabalhabilidade foi baixa independente da granulometria com o tempo máximo de aproximadamente 16 min quando permaneceu na estufa durante 17h. Esse tempo é muito alto para ter uma eficiência energética no processo de reciclagem do gesso.

$\mathrm{Na}$ temperatura de $136^{\circ} \mathrm{C}$ o gesso reciclado durante $1 \mathrm{~h}$ apresentou uma trabalhabilidade acima de 100 min em todas as granulometrias estudadas. De acordo com o difratograma de Raios X (Figura 4) em 1h de calcinação ainda tem a presença de dihidrato no gesso reciclado $(0,30 \mathrm{~mm})$ indicando que esse tempo não foi suficiente para conversão do resíduo de gesso em hemihidrato para permitir um tempo de pega adequado durante a hidratação. A granulometria de $0,075 \mathrm{~mm}$ mostrou pequenas trabalhabilidades a partir de $6 \mathrm{~h}$ de calcinação. Provavelmente devido à trabalhabilidade tender a diminuir com a redução da granulometria, pois ocorre o aumento da atividade cinética da reação de hidratação [36].

$\mathrm{Na}$ calcinação realizada a $150^{\circ} \mathrm{C}$ utilizando a granulometria de $0,075 \mathrm{~mm}$ houve trabalhabilidade a partir de $4 \mathrm{~h}$, nas granulometrias de 0,3 e $2 \mathrm{~mm}$ em $1 \mathrm{~h}$ a trabalhabilidade foi superior a $100 \mathrm{~min}$. Nos demais tempos de calcinação $(2 \mathrm{~mm})$ mostrou uma tendência a aumentar a trabalhabilidade.

O resíduo de gesso apresentou uma granulometria maior que o gesso comercial (Tabela 2), logo a taxa de hidratação aumenta com a redução do tamanho das partículas devido a maior área específica do grão, pois o processo de hidratação inicia em sua superfície externa [37, 25].

$\mathrm{Em} 170^{\circ} \mathrm{C}$ de forma geral o gesso reciclado com $0,3 \mathrm{~mm}$ apresentou as maiores trabalhabilidades quando comparado com as demais granulometrias. Foi obtida a trabalhabilidade média acima de 15 min a partir de $4 \mathrm{~h}$ de calcinação em todas as granulometrias estudadas.

Observou-se uma pequena perda na trabalhabilidade no gesso recliclado $(0,30 \mathrm{~mm})$ a 136,150 e $170^{\circ} \mathrm{C}$ durante 8 e $17 \mathrm{~h}$, sem haver variações relevantes nas propriedades mecânicas (Tabela 4), comportamento semelhante foi reportado em um gesso submetido à reciclagem a $150^{\circ} \mathrm{C}$ [12]. Esses parâmetros e os tempos de calcinação na temperatura de análise térmica foram selecionados para avaliação da cinética de hidratação. 
A Figura 7 apresenta as curvas de hidratação para o gesso reciclado em diferentes parâmetros. Foi selecionada a granulometria intermediária $(0,30 \mathrm{~mm})$ para avaliação da cinética de hidratação e das propriedades mecânicas.

A hidratação do gesso pode ser monitorada por meio da calorimetria [3]. A partir das curvas da cinética de temperatura da pasta de gesso, foi possível medir a temperatura máxima atingida durante a hidratação do gesso e os tempos inicial e final de pega [38].

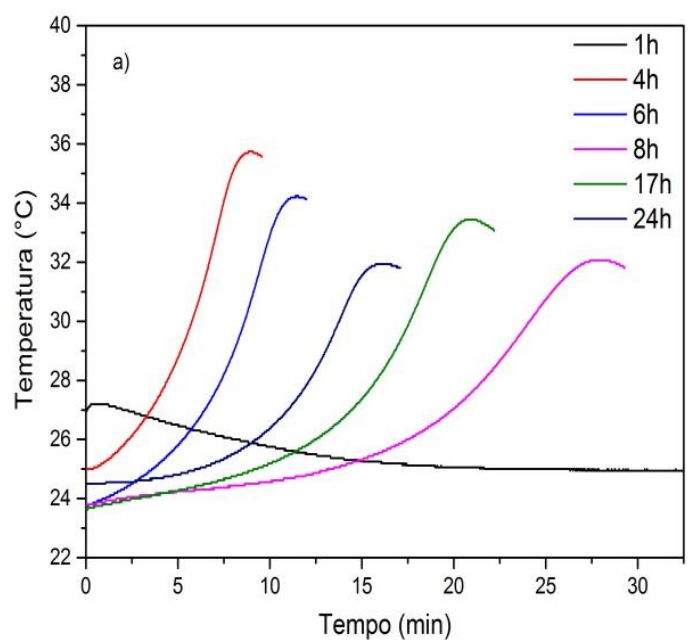

(a)

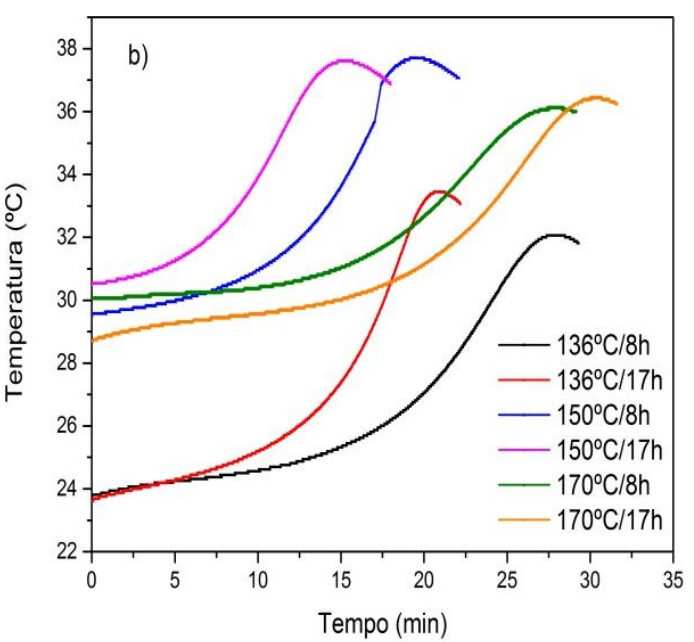

(b)

Figura 7: Curvas de hidratação: a) resíduo de gesso calcinado a temperatura da análise térmica $136^{\circ} \mathrm{C}$ em diferentes tempos e b) resíduo de gesso calcinado a 136,150 e $170^{\circ} \mathrm{C}$ durante 8 e $17 \mathrm{~h}$.

Na Figura 7-a o gesso reciclado durante uma 1h de calcinação a pasta não atingiu a pega até 35 min de ensaio. O tempo de início de pega também é influenciado pelo tempo de calcinação do gesso [26]. Os demais tempos apresentaram inicio de pega semelhante e o final da pega não apresentou uma tendência com o aumento do tempo de calcinação, sendo a permanência na estufa de $8 \mathrm{~h}$ o maior tempo de fim de pega. Na Figura 7-b as curvas apresentaram comportamento semelhante de acordo com a temperatura. Nas temperaturas de 136 e $150^{\circ} \mathrm{C}$ o tempo de $8 \mathrm{~h}$ de calcinação apresentou um final de pega maior que $17 \mathrm{~h}$, indicando um ganho em um menor tempo de estufa. Enquanto que na temperatura de $170^{\circ} \mathrm{C}$ os tempos de pega são similares independentes do tempo de calcinação.

Vários fatores podem influenciar a reação de hidratação do gesso, e consequentemente o tempo de pega como a relação água/gesso, a presença de impurezas ou grãos de areia conforme mostrado na análise química (Tabela 1), podem atuar como núcleos de cristalização, acelerando as reações de hidratação [38, 10]. resistência à compressão e a dureza do gesso reciclado (Tabela 4). A resistência a compressão apresentou um média de 5,43 MPa quando calcinado por $8 \mathrm{~h}$ e de 6,33 MPa durante17h. Os melhores resultados para a resistência compressão foram obtidos na temperatura de $136^{\circ} \mathrm{C}(17 \mathrm{~h})$ e $150^{\circ} \mathrm{C}(8 \mathrm{~h})$. Em estado endurecido, as pastas de gesso reciclado geralmente mostram uma perda de resistência mecânica, dependendo do conteúdo de substituição nas misturas, o tamanho das partículas e o teor de impurezas [6]. A presença de poros na microestrutura relacionada com o processo de secagem da quantidade de água utilizada na mistura, também pode influenciar na redução das propriedades mecânicas [39].

O gesso reciclado apresentou uma dureza média de $12,71 \mathrm{~N} \cdot \mathrm{mm}^{-2}$, inferior ao valor mínimo de $20 \mathrm{~N} \cdot \mathrm{mm}^{-2}$ conforme a norma NBR 13207 [16].

Tabela 4: Propriedades mecânicas do gesso reciclado.

\begin{tabular}{|c|c|c|c|}
\hline TEMPERATURA DE CALCINAÇÃO $\left({ }^{\circ} \mathrm{C}\right)$ & TEMPO (h) & $\begin{array}{c}\text { RESISTÊNCIA À } \\
\text { COMPRESSÃO (MPa) }\end{array}$ & DUREZA $\left(\mathbf{N} \cdot \mathbf{m m}^{-2}\right)$ \\
\hline \multirow{2}{*}{136} & 8 & $4,41 \pm 0,37$ & $11,19 \pm 1,18$ \\
\hline & 17 & $6,90 \pm 0,55$ & $15,40 \pm 2,32$ \\
\hline \multirow{2}{*}{150} & 8 & $6,92 \pm 0,23$ & $12,93 \pm 0,86$ \\
\hline & 17 & $5,78 \pm 0,24$ & $11,76 \pm 0,28$ \\
\hline \multirow{2}{*}{170} & 8 & $4,96 \pm 0,16$ & $12,11 \pm 1,02$ \\
\hline & 17 & $6,31 \pm 0,53$ & $12,87 \pm 1,92$ \\
\hline
\end{tabular}




\section{CONCLUSÕES}

As propriedades do gesso reciclado obtido a partir das reações de calcinação do resíduo de gesso sofreu influência dos parâmetros avaliados: a granulometria, a temperatura e o tempo de desidratação. As principais fases formadas no gesso reciclado foram afetadas pelo tempo de calcinação: o sulfato de cálcio hemihidratado, sulfato de cálcio dihidratado, anidrita e calcita.

A trabalhabilidade não mostrou uma tendência clara de comportamento independente da granulometria, tempo e temperatura de calcinação. Os melhores resultados para a resistência compressão foram obtidos na temperatura de $136^{\circ} \mathrm{C}(17 \mathrm{~h})$ e $150^{\circ} \mathrm{C}(8 \mathrm{~h})$. O gesso reciclado apresentou uma dureza média de $12,7 \mathrm{~N} \cdot \mathrm{mm}^{-2}$, inferior ao valor mínimo esperado na norma. A reciclagem do resíduo de gesso pode contribuir com a redução dos resíduos e obtenção de melhores propriedades do produto final com a utilização em maior escala do gesso reciclado.

\section{AGRADECIMENTOS}

Os autores agradecem a Coordenação de Aperfeiçoamento de Pessoal de Nível Superior (CAPES), pelo apoio financeiro concedido por meio da bolsa de pós-doutorado (PNPD).

\section{BIBLIOGRAFIA}

[1] SHIROMA, L., CAMARINI, G., BERALDO, A. L., "Effect of wood particle treatment on the properties of gypsum plaster pastes and composites", Revista Matéria, v. 21, n. 4, pp. 1032-1044, Jul. 2016.

[2] CAMARINI, G., MILITO, J.A., "Gypsum-cement hemihydrate blends to improve renderings durability", Construction and Building Materials, v. 25, pp. 4121-4125, 2011.

[3] BERNHOEFT, L.F., GUSMÃO A.D., TAVARES, Y.V.P., "Influência da adição de resíduo de gesso no calor de hidratação da argamassa de revestimento interno", Ambiente Construído, v. 11, n. 2, pp. 189-199, Abr./Jun. 2011.

[4] ALMEIDA, K.S., SOARES, R.A.L., MATOS, J.M.E., "Efeito de resíduos de gesso e de granito em produtos da indústria de cerâmica vermelha: revisão bibliográfica", Revista Matéria, v.25, n.1, 2020.

[5] GERALDO, R.H., PINHEIRO, S.M.M., SILVA, J.S., et al., "Gypsum plaster waste recycling: A potential environmental and industrial solution", Journal of Cleaner Production, v. 164, pp. 288-300, Jun. 2017.

[6] SANTANA, C.V., PÓVOAS, Y.V., SILVA, D.G.C., et al., "Recycled gypsum block: development and performance", Ambiente Construído, v. 19, n. 2, pp. 45-58, Abr./Jun. 2019.

[7] CAMARINI, G., PINTO, M.C.C., MOURA, A.G., et al., "Effect of citric acid on properties of recycled gypsum plaster to building componentes", Construction and Building Materials, v. 124, pp. 383-390, Jul. 2016.

[8] MEDINA, N.F., HERNÁNDEZ-OLIVARES, F., ARROYO, X., et al., "Characterization of a more sustainable cement produced with recycled drywall and plasterboards as set retarders", Construction and Building Materials, v. 124, pp. 982-991, Set. 2016.

[9] PAPAILIOPOULOU, N., GRIGOROPOULOU, H., FOUNTI, M., "Techno-economic impact assessment of recycled gypsum usage in plasterboard manufacturing", Journal of Remanufacturing, pp.1-27, Oct. 2018.

[10] TAVARES, Y.V.P., JÚNIOR, A.C.L., SCHMITZ, I.B.T.A., et al., "Reaproveitamento do resíduo de gesso na execução de revestimento interno de vedação vertical", Ambiente Construído, v. 10, n. 11, pp. 103119, Jan./Mar. 2010.

[11] ZHIXIN, L.I., JIAHUI, P., XINGXING, Q.I.U., "Effect of Different Ways of STPP Retarder Addition on Properties of Recycled Gypsum”, Journal of Wuhan, v. 32, n. 5, pp. 1125-1131, Oct. 2017.

[12] ROSSETO, J.R.M., CORREIA L.S., GERALDO, R.H., et al., "Gypsum plaster wastw recycling: analysis of calcination time", Key Engineering Materials, v. 668, pp. 312-321, Out. 2015.

[13] PEDREÑO-ROJAS, M.A., FLORES-COLEN, I., BRITO, J., et al., "Influence of the heating process on the use of gypsum wastes in plasters: Mechanical, thermal and environmental analysis", Journal of Cleaner Production, v. 215, pp. 444-457, Jan. 2019.

[14] INAZUMI, S., SANO, H., YAMADA, M., "Estimation of gypsum hemihydrate content in recycled gypsums derived from gypsum boards", Journal of Material Cycles and Waste Management, v. 18, pp. 168-176, Oct. 2016.

[15] JACQUES, S.D.M., GONZALEZ-SABORIDO, A., LEYNAUD, O., et al., "Structural evolution during 
the dehydration of gypsum materials", Mineralogical Magazine, v. 73, n. 3, pp. 421-432, Jun. 2009.

[16] EMBRAPA - Manual de métodos e análise de solos, $3^{\mathrm{a}}$ Edição, 2017.

[17] Associação Brasileira de Normas Técnicas - ABNT NBR 12127: Gesso para construção - determinação das propriedades físicas do pó, Rio de Janeiro, 2017.

[18] Associação Brasileira de Normas Técnicas - ABNT NBR 13207: Gesso para construção civil. Rio de Janeiro, 1994.

[19] ANTUNES, R.P.N., Estudo da Influência da Cal Hidratada nas Pastas de Gesso, Dissertação de M. Sc, Escola Politécnica, UFSP, São Paulo, 1999.

[20] Associação brasileira de normas técnicas - ABNT NBR 12129: gesso para construção Determinação das propriedades mecânicas: Rio de Janeiro, 1991c.

[21] LÊLA, L.A. Desenvolvimento de dispositivo para determinar dureza mecânica em gesso segundo NBR 12129. Trabalho de conclusão de curso. Engenharia Mecânica - UNIVASF, 2017.

[22] NGERNCHUKLIN, P., YONGPRADERM, N., BOONRUANG, A., et al., "Upgrading of Waste Gypsum for Building Materials", Key Engineering Materials, v. 766, pp. 211-216, Abr. 2018.

[23] BARBOSA, A.A., FERRAZ, A.V., SANTOS, G.A., "Caracterização química , mecânica e morfológica do gesso $\beta$ obtido do pólo do Araripe", Cerâmica, v. 60, pp. 501-508, Out./ Nov./ Dez. 2014.

[24] CARVALHO, M.T.M., LEES, M.I.M., TUBINO, R.M.C., "TG and DSC studies on plaster residues as recycled material", Journal of Thermal Analysis and Calorimetry, v. 91, n. 2, pp. 621-625, 2008.

[25] ERBS, A., NAGALLI, A., MYMRINE, V., et al., "Determinação das propriedades físicas e mecânicas do gesso reciclado proveniente de chapas de gesso cartonado", Cerâmica, v. 61, pp. 483-487, Abr./ Jun. 2015.

[26] SAVI, O., Produção de placas de forro com a reciclagem do gesso, Dissertação de M.Sc, UEM, Maringá, PR, Brasil, 2012.

[27] Associação Brasileira de Normas Técnicas - ABNT NBR 12128: Gesso para a construção civil: determinação das propriedades físicas da pasta de gesso, Rio de Janeiro, 2017.

[28] GOLEŽ, M., POGAČNIK, Ž., MLADENOVIĆ, A., "Laboratory-prepared lime-gypsum mixtures based on the know-how of traditional technology", Journal of Cultural Heritage, v. 32, pp. 38-43, Mar. 2018.

[29] NITA, C., PILEGGI, R.G., CINCOTTO, M.A., et al., "Estudo da reciclagem do gesso de construção", In: X Encontro nacional de tecnologia do ambiente construído, I conferência latino-americana de construção sustentável, São Paulo-SP, Jul. 2004.

[30] JO, D., LEONARDO, R.S., CARTLEDGE, F.K., et al., "Gypsum content determination in Portland cements by thermogravimetry", Journal of Thermal Analysis and Calorimetry, v. 123, n. 2, pp. 1053-1062, Oct. 2016.

[31] ERBS, A., NAGALli, A., CARVALHO, K.Q., et al., "Properties of recycled gypsum from gypsum plasterboards and commercial gypsum throughout recycling cycles. Journal of Cleaner Production, v. 183, pp. 1314-1322, Feb. 2018.

[32] MOISSET, J., "Review of the Main Process Used for Plaster Products" Gypsum Fair 97, ed. Technological Institute of Pernambuco - ITEP, Brazil 1997.

[33] PINHEIRO, S.M.M., CAMARINI, G., "Characteristics of Gypsum Recycling in Different Cycles", International Journal of Engineering and Technology, v. 7, n. 3, pp. 215-218, Jun. 2015.

[34] MELO, K.K.S., LIMA, A.P.C., SANTANA, M.C., et al., "Caracterização química e mineralógica dos resíduos da mineração de gipsita no semiárido pernambucano”, Holos, v. 06, pp. 194-200, Nov. 2017.

[35] PINHEIRO, S.M.M., CAMARINI, G., VIEIRA, G.L., "Utilização do resíduo de gesso de construção na produção de componentes", In: V Encontro Nacional Sobre Aproveitamento de Resíduos na Construção (ENARC), pp. 1-15, Fortaleza-CE, Ago. 2017.

[36] BARDELlA, P.S., Análise das Propriedades de Pastas de Gesso de Construção Reciclado, Tese D. Sc, UNICAMP, Campinas, SP, Brasil, 2011.

[37] SHIROMA, L., CAMARINI, G., BERALDO, A.L., "Effect of wood particle treatment on the properties of gypsum plaster pastes and composites", Revista Matéria, v. 21, n. 4, pp. 1032-1044, Jul. 2016. 
[38] ANTUNES, R. P. N., JOHN, V. M., O Conceito de Tempo Útil das Pastas de Gesso. São Paulo: Escola Politécnica da Universidade de São Paulo, 2000. Boletim técnico.

[39] VILLELA, L.S., CASTRO, E.D., JÚNIOR, L.M., et al., "Desempenho físico-mecânico de compósitos à base de gesso reforçados com embalagens multicamadas trituradas", Revista Matéria, v. 25, n. 3, Jan. 2020.

\section{ORCID}

Pâmela Bento Cipriano

https://orcid.org/0000-0002-3013-3854

Clebiano Santos Sá

https://orcid.org/0000-0002-6728-471X

Tássila Saionara Gomes Galdino

https://orcid.org/0000-0002-6190-3735

Andréa de Vasconcelos Ferraz

https://orcid.org/0000-0001-8043-1414 